\title{
Longitudinal Effects of Integrated Treatment on Alcohol Use for Persons with Serious Mental Illness and Substance Use Disorders
}

\author{
Sandra E. Herman, Ph.D. \\ Kenneth A. Frank, Ph.D. \\ Carol T. Mowbray, Ph.D. \\ Kurt M. Ribisl, Ph.D. \\ William S. Davidson II, Ph.D. \\ Bonnie BootsMiller, Ph.D. \\ Lisa Jordan, Ph.D. \\ Arnold L. Greenfield, Ph.D. \\ David Loveland, M.A. \\ Douglas A. Luke, Ph.D.
}

\begin{abstract}
A randomized experimental design was used to assign participants to an integrated mental health and substance use treatment program or to standard hospital treatment. A multilevel, nonlinear model was used to estimate hospital treatment effects on days of alcohol use for persons with serious mental illness and substance use disorders over 18 months. The integrated treatment program had $a$ significant effect on the rate of alcohol use at 2 months postdischarge, reducing the rate of use by $54 \%$. Motivation for sobriety at hospital discharge, posttreatment self-help attendance, and social support for sobriety were also found to reduce the rate of use during the follow-up period. Implications for mental health treatment and aftercare support are discussed.
\end{abstract}

Program models for treating persons with serious mental illness and substance use disorders (SMI-SD) generally involve hybrid or integrated services. ${ }^{1.3}$ These programs emphasize the need to recognize stages of treatment and the importance of persuasion and engagement. ${ }^{3.6}$ Studies of the effectiveness of integrated inpatient programs have indicated mixed results. Some studies have found improved community functioning. ${ }^{7 \cdot 13}$ Yet, others have been unable to identify

Address correspondence to Sandra E. Herman, Ph.D., Program Evaluation Specialist, Services Research Unit, Mental Health and Substance Abuse Services, Department of Community Health, Lewis Cass Building, 320 S. Walnut, Lansing, MI 49813; e-mail: herman@state.mi.us.

Kenneth A. Frank, Ph.D., is an associate professor in the Department of Education at Michigan State University.

Carol T. Mowbray, Ph.D., is a professor in the School of Social Work at the University of Michigan.

Kurt M. Ribisl, Ph.D., is an assistant professor in the Department of Health Behavior and Health Education at the University of North Carolina, School of Public Health.

William S. Davidson, II, Ph.D., is a professor in the Department of Psychology at Michigan State University. 
posthospitalization treatment effects. ${ }^{14-17}$ A review by Drake et al.$^{18}$ of 36 studies of the effectiveness of integrated treatment programs, including inpatient intensive integrated treatment and comprehensive integrated dual disorder treatment, noted mixed results from many of these studies but concluded that there is encouraging evidence that these programs do engage persons with SMI-SD and help them reduce their substance use. This review also points out that outcomes related to posttreatment hospital use, psychiatric symptoms, and other domains are less consistent.

A number of these studies have looked for direct effects of SMI-SD treatment on later psychiatric and substance use outcomes. These studies have not, however, explicitly modeled the effects of proximal or suboutcomes of treatment (e.g., changes in motivation, attitudes, behaviors) ${ }^{19}$ on longitudinal substance use and functioning. Several authors have emphasized the need for after-care services following hospitalization ${ }^{17,20-22}$ and have examined the longitudinal effects of treatment. They have not explicitly modeled the effects of posttreatment environmental variables (such as aftercare services, use of self-help groups, and support for sobriety from family and friends) on treatment outcomes over time. In this study, the extant literature is moved forward by using growth models to examine the moderating effects of personal characteristics and the mediating effects of proximal outcomes on the relation of treatment and changes in use of alcohol. The theoretical model of Finney and Moos, ${ }^{19,24}$ was used to develop the growth models tested in this study. This model (Figure 1) hypothesizes that prior individual characteristics will moderate the effects of SMI-SD inpatient treatment on longitudinal community outcomes. It also hypothesizes that proximal treatment outcomes (e.g., motivation for sobriety) and posttreatment environmental characteristics are intervening variables in the causal processes that occur between treatment and observations of long-term outcomes. Consumers' differential posttreatment functioning may be associated with differences in their immediate treatment outcomes (e.g., their motivation for sobriety) and their posttreatment environments (e.g., rehospitalization, use of community based treatments, self-help attendance, or social support for sobriety).

The individual characteristics of people diagnosed with SMI-SD describe them as younger, more often male, less compliant with treatment, and engaged in more violent or criminal behavior than persons with only a diagnosis of mental illness. ${ }^{25-26}$ However, conflicting results are reported for community functioning, substances of choice, and the role of personal characteristics in treatment of persons with SMI-SD. ${ }^{12,27-31}$ The conflicting results of previous studies regarding the role of gender, age, ethnicity, diagnosis, and treatment history suggest that one or more of these variables may moderate the effects of treatment.

Posttreatment environmental characteristics are another set of variables that may influence posttreatment outcomes. These variables cover a broad range of community-living experiences, work, social support, formal treatment alternatives (e.g., community-based mental health and substance use services), and informal treatment in the form of self-help organizations and groups. ${ }^{3.29}$ In this study, incarceration and rehospitalization were used as indicators of restrictions in the posttreatment environment that may reduce access and use of substances. ${ }^{29}$

Self-help interventions using 12-step or Alcoholics Anonymous (AA) models are now considered a core component of integrated treatment programs for persons with SMI-SD. ${ }^{30-34}$ There is evidence that a strength of integrated treatment programs is their preparation of patients to successfully participate in self-help groups. ${ }^{19,30}$ The literature on AA outcomes for persons without mental illness

Bonnie BootsMiller, Ph.D., is at the Center for Practice Management and Outcome Research, VA Ann Arbor Health Care System.

Lisa Jordan, Ph.D., is an assistant professor in the Department of Psychology at the University of Maryland-Baltimore County.

Amold L. Greenfield, Ph.D., is a program evaluation specialist at the Services Research Unit, Mental Health and Substance Abuse Services, Department of Community Health.

David Loveland, M.A., is a research assistant in the Department of Psychology at Michigan State University.

Douglas A. Luke, Ph.D., is an associate professor at St. Louis University, School of Public Health. 


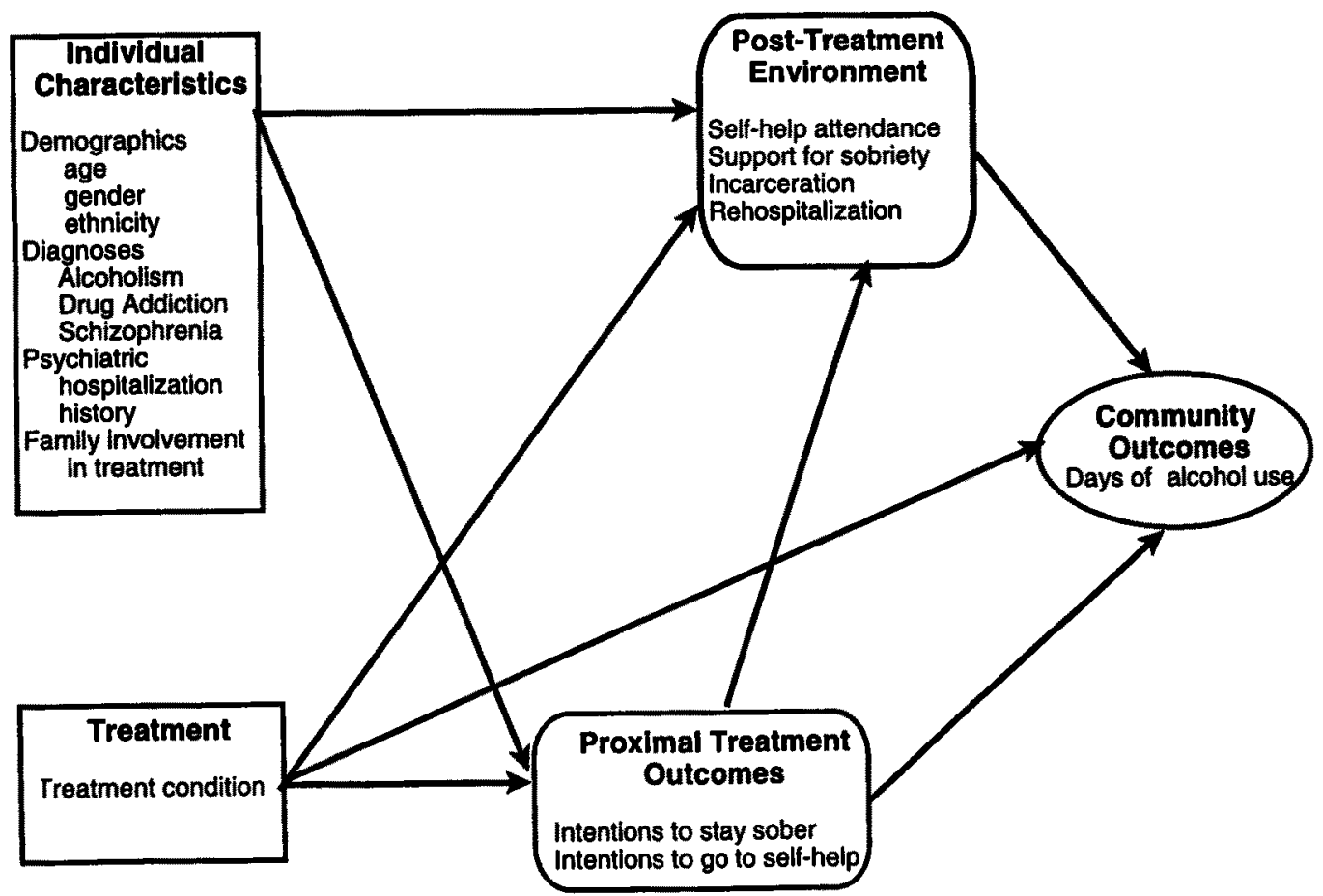

suggests that 12-step groups can play a significant role in the recovery from substance use problems. These groups provide environmental and social supports for recovery and abstinence. Johnsen and Herringer $^{35}$ found that attendance at AA or Narcotics Anonymous (NA) meetings was significantly related to posttreatment abstinence, whereas other social factors did not independently predict abstinence. Finney et al. ${ }^{19}$ suggest that the addition of 12 -step support groups to outpatient aftercare services may result in improved long-term outcomes because the types of proximal treatment outcomes produced by 12-step models support sobriety. Drake et al. ${ }^{18,36}$ have noted the need to motivate persons with SMI-SD to participate in 12-step type groups. The support provided by self-help groups is critical for persons with SMI-SD who may lack the social skills needed to replace substance-using peers with sober peers, have difficulty structuring their time and activities, ${ }^{33}$ and need the same type of support and benefits begun in formal treatment programs. ${ }^{33}$

The posttreatment environment domain also includes a variety of social resources, such as general social support from family and social network members and support for staying sober from network members. ${ }^{33,37}$ These resources can provide significant support and reinforcement to individual commitment to change to a healthier lifestyle and resist relapse. ${ }^{3,33,37,38}$ The person's family and social network play critical roles in supporting and reinforcing reductions in the use of alcohol and drugs. ${ }^{38}$ Studies in the alcohol field have identified positive association between social support and reduction of alcohol-related problems. ${ }^{39,40}$ Furthermore, epidemiological studies have shown that for persons with any mental or addictive disorder, a large proportion (37\%) of requests for help are to support networks composed of friends, relatives, and self-help groups. ${ }^{41}$ 
Motivation for change is a pivotal variable and a key proximal outcome for dual treatment programs. ${ }^{3.6}$ The literature on alcohol treatment suggests that motivation for change is a key variable in whether treatment is effective. ${ }^{42}$ Finney et al. ${ }^{19}$ compared motivational and belief variables for persons in programs using 12-step models to persons in programs using cognitive-behavioral models and found significantly greater change in motivation for programs using 12-step-type models. Finney et al. argue that in 12-step-type programs, changes in motivation are the proximal outcomes. Research on processes of change for both persons with serious mental illness and persons with substance abuse disorder has often emphasized the importance of motivation for change and treatment goals. ${ }^{43}$ The theory on which most specialized SMI-SD programs are based also argues that changing the person's motivation to refrain from using substances is an immediate outcome of treatment and a prerequisite for preventing relapse. ${ }^{4,44,45}$ Thus, the attitudes that persons with SMI-SD diagnoses hold about mental illness, substance abuse, and addiction at the conclusion of formal treatment should play an important role in their patterns of community functioning. Drake et al ${ }^{18,36}$ noted in their conclusions that motivational interventions are necessary to prepare persons with SMI-SD for abstinence-oriented interventions. Motivation, however, is a little-examined dimension in research on persons with SMI-SD but should influence posttreatment outcomes.

In this study, the impact of an inpatient-integrated mental health and substance use treatment program was assessed by comparing the days of alcohol use over 18 months by persons who received the dual treatment with those who did not. Previous analyses of outcomes at discharge (proximal outcomes) had indicated that persons receiving SMI-SD treatment were more motivated to stay sober and to go to self-help groups after discharge. ${ }^{46}$ The effects of the latter factors were explicitly modeled on growth curves models of individual change over time accounting for the treatment received and other covariates.

\section{Method}

\section{Procedures}

Sample recruitment occurred over a 12-month period during which all persons newly admitted to the hospital were screened to identify those who met criteria for mental illness and substance abuse. Eligibility required a positive screening for alcohol or drugs (based on the 28-item Michigan Alcoholism Test ${ }^{47}$ modified to include alcohol and drug use), clinical determination of substance abuse problems, and willingness of the consumer to accept admission to the Mental Health Chemical Dependence (MHCD) program. Persons who displayed unmanageable behavior requiring extensive seclusion and restraint were excluded (estimated to be $10 \%$ of those eligible). During recruitment, both treatment programs were described to the person along with the purpose and procedures of the study. Informed consent was obtained from each participant prior to treatment assignment. Assignment to treatment condition was randomized within gender and order of enrollment with a ratio of 2:1 assignment to the MHCD program (experimental) to the standard short-term treatment wards (STW) (control). Participants were interviewed during their first week in the study, 4 weeks later or at discharge (whichever occurred first), and at 2,6,10,14, and 18 months postdischarge by interviewers hired by the research project. The study sample contained 485 members. Retention rates for follow-up interviews at $2,6,10,14$, and 18 months were, respectively, $86.7 \%, 85.6 \%, 85.1 \%, 84.5 \%$, and $88.4 \%{ }^{48}$ Of the 485 sample members, 429 had at least one postdischarge interview, with $76 \%$ having all five postdischarge interviews. Analyses presented here are based on these 429 participants.

\section{Setting}

The study was conducted at a JCAHO-accredited state psychiatric hospital serving a large urban catchment area in the Midwest. The hospital's MHCD program provided specialized, coeducational 
treatment on two 30-bed wards that had a 3:2 ratio of male to female beds and served as the experimental condition. STWs were used as the control condition. The MHCD program had mental health staff trained in substance abuse treatment and had a 1:6 staff-to-patient ratio. STW mental health staff were not trained in substance abuse treatment, and these wards had 1:8 staff-to-patient ratio.

The MHCD program was based on a staged theoretical model. ${ }^{2,4,18,24,46}$ These stages of treatment may overlap and are not necessarily sequential for each individual. Stage 1 was the stabilization of acute psychiatric and physical symptoms for both the STW and MHCD programs and included medical and psychiatric assessment and screening for substance abuse. Stage 2 engaged the consumer in the treatment process using individual, group, and activity therapy. Additionally, the MHCD program provided educational lectures, AA and/or NA groups, family education sessions, and gender-specific support groups to engage participants. The MHCD program focused on persuasion and awareness in stage 3 through the use of 40 formal educational lectures that covered the disease concept of addiction, physiological effects of drugs, HIV/AIDS prevention health issues, recovery and relapse, and mental illness concepts and treatment issues. The lectures were intended to provide information, change consumers' attitudes about substance use, and increase motivation for program involvement and recovery. The STW program had no similar treatment component. Stage 4 involved active treatment through individual and group psychotherapy and activity therapy for both conditions. The MHCD program provided a minimum of one hour per week of individual therapy and one hour per day (five hours per week) of group therapy compared with a half hour per week of individual and one hour per week of group therapy in STW. The MHCD mental health treatment also addressed drug and alcohol addiction problems, and therapy was focused on reducing denial, enhancing coping skills, and improving interpersonal relationships. Furthermore, the MHCD program required attendance at two AA and/or NA meetings per week, while the STW did not emphasize substance use treatment although consumers could elect to attend AA and/or NA meetings. Finally, stage 5 focused on relapse prevention through the discharge-planning process and was similar for both groups. The MHCD program additionally emphasized strategies for remaining clean and sober and identified AA and NA groups in the community to which the consumer was returning.

The MHCD group stayed, on average, 20 days longer in the hospital than the STW group (51.4 days vs. 31.1 days). Although the two groups were comparable on entry, difference in length of stay could produce differences in levels of behavior and attitudes on discharge. The multilevel models used to assess the treatment effect account for the treatment effect at two months postdischarge as well as treatment effect on rate of decline from admission to two months postdischarge. As a rate, this latter effect is independent of the length of stay. At discharge, the MHCD group had greater knowledge of substance use issues and 12-step programs and expressed stronger motivation to control their substance use, remain sober, and attend 12-step groups after hospitalization. ${ }^{46}$ These variables represent the proximal outcomes for treatment and provide some evidence that cross contamination of the experimental and control groups was not a major factor.

\section{Sample}

Descriptive variables for 429 participants are presented in Table 1. Participants were distributed similarly in MHCD and STW conditions. No significant differences between the groups were found at admission in demographics, previous treatment history, importance of treatment to the person, or functioning before hospitalization. The majority of participants were males in their mid-30s. Two-thirds of the sample had one or more prior psychiatric hospitalizations. Psychiatric and substance use diagnoses also are presented in Table 1. Almost two-thirds of the participants had diagnoses indicating serious mental illness (schizophrenia, organic mood disorders, major depression, or bipolar depression). Nearly three-quarters of the participants had diagnoses related to alcohol use. Diagnoses related to cocaine use were the most frequent nonalcohol drug use diagnoses. 
Table 1

Sample Characteristics (in percentages)

Proportion male

Ethnicity

African American

White

Treatment assignment

Experimental

Control

Mean age in years $(S D)$

DSMIII-R diagnosis

Mental illness

Schizophrenic disorders

Organic mood disorders

Mild affective disorders

Adjustment disorder

Major depression

Bipolar depression

Antisocial personality

Substance abuse ${ }^{\mathrm{a}}$

Alcohol dependence

Alcohol abuse

Cocaine dependence

Cocaine abuse

Cannabis dependence

Cannabis abuse

NOTE: $N=429$.

a. Multiple diagnoses were permitted.

\section{Analysis Model}

The Addiction Severity Index (ASI) ${ }^{49-51}$ was used as the major data collection tool.* Scale scores for the ASI are constructed to range from 0 (no problem) to 1 (high problem level). However, the ASI metric is not clearly defined, nor are these scales necessarily interval level. These issues are of concern whenever using the ASI in a statistical analysis. However, they are of special concern when modeling change over time, as change must be based on an interval scale measure. ${ }^{52}$

To address these concerns regarding the scale of the ASI, examinations were conducted of the distribution of the ASI alcohol scores at each time point, as well as residuals from linear regression of the ASI regressed separately at each time point on variables to be used in the growth models. Although the ASI alcohol scores were approximately normally distributed on intake (reflecting its validity as a measure used for clinical diagnosis), the distributions became increasingly positively skewed with time (i.e., there were many subjects who scored on the lowest end and only a few who scored on the highest end, leaving a long positive tail). Consequently, the ASI is not an optimal mea-

*Modifications to the Addiction Severity Index recommended by the authors for use with persons with serious mental illness and substance use disorders were used. ${ }^{51}$ In this study, internal consistency coefficients (average .70, range .56 to .86 ) were found that compared favorably to reliability figures for a dual diagnosis sample reported by Hodgins and El-Guebaly. 
sure for modeling change over time. In particular, at later time points, decreases on the lower end of the scale may be much more difficult to achieve than decreases on the higher end of the scale.

To address this concern, two modifications of the ASI score were considered. First, the logit transformation $(\log [\mathrm{ASI} /(1-\mathrm{ASI})]$ ) was used to correct the skew. This improved the distribution of the residuals from each time point somewhat. When the logit of ASI alcohol scores was modeled, the substantive interpretations were the same as those based on the analyses to be reported here.

The second approach was to deconstruct the ASI alcohol score and focus the analysis on the number of days of alcohol use in the month prior to each interview. This outcome is a count, and consequently a Poisson sampling model with a log-link function was used at level 1 of the analysis (the Poisson sampling model is intended for counts and can accommodate skewed distributions in which many people have low counts).* The number of days of alcohol use also provides a behaviorally based measure with an easily understood metric and is an interval scale (e.g., the difference between 1 and 5 is the same as the difference between 21 and 25 ; both are a change of 4 units). The results from this analysis are reported here. The parameters of this latter analysis are easier to interpret, representing the effect on the number of days of alcohol use in the past 30 days.

Building the model for change in days of alcohol use over time began by considering a standard regression model for days of alcohol use (DAU) at time $t$ as a function of time:

$$
\log \left(\mathrm{DAU}_{t}\right)=\beta_{0}+\beta_{1} \text { time }_{1} \text {. }
$$

Time was measured as months relative to discharge, which accommodated the fact that different people were measured at different intervals and that different people were placed in the program at different times of the year. The effect of time may have been different during the period of the treatment than during the extended posttreatment period. In particular, since the participants were in crises when they entered the treatment, and since both treatment and control involved hospitalization, the most dramatic decrease in days of alcohol use might be expected to occur during the interval right around treatment. Visual examination of the data supported this expectation (Figure 2). Therefore, the effect of time was separated into two pieces:

$$
\begin{gathered}
\log (\mathrm{DAU},)=\beta_{0}+\beta_{1} \text { time from admission to } 2 \text { months } \\
\text { postdischarge } e_{t}+\beta_{2} \text { time } 2 \text { months postdischarge through end of study, }
\end{gathered}
$$

Here, $\beta_{1}$ represents the effects of time in the first interval, and $\beta_{2}$ represents the effects of time in the second interval.

In this model, time points were nested within people, and the effects of people must be accounted for in the model. Consequently, the assumption made for statistical inference that the error terms were independent was not likely to be satisfied, because any two error terms associated with the same person were likely to be positively correlated (see Bryk and Raudenbush, chap. $6^{53}$ ). To address the nested nature of the data, the model (equation 2) was extended to a multilevel framework:

At level 1 (time points): $\quad \log \left(\mathrm{DAU}_{i t}\right)=\beta_{0 \mathrm{i}}+\beta_{l i}$ time from admission to 2 months

postdischarge $_{i t}+$

$\beta_{2 i}$ time 2 months postdischarge through end of study $y_{i t}$

\footnotetext{
*The log-link function is used to ensure that predictions are constrained to be within a given interval; for example, in this analysis, the event rate is constrained to be nonnegative because it is a count. The log of the event rate can take any real value (negative or positive), while the event rate can only be nonnegative. Thus, the predicted values for this analysis are predicted $\log$-event rates in which a negative value indicates a decline and a positive value indicates an increase in the event rate. The $\log$-event rate can be converted to event rates by computing: event rate $=\operatorname{exponential}\left(n_{i j}\right)$, where $n_{i j}=\log$ of the event rate.
} 
Figure 2

Mean Days of Alcohol Use Reported at

Admission and Follow-Up Interviews

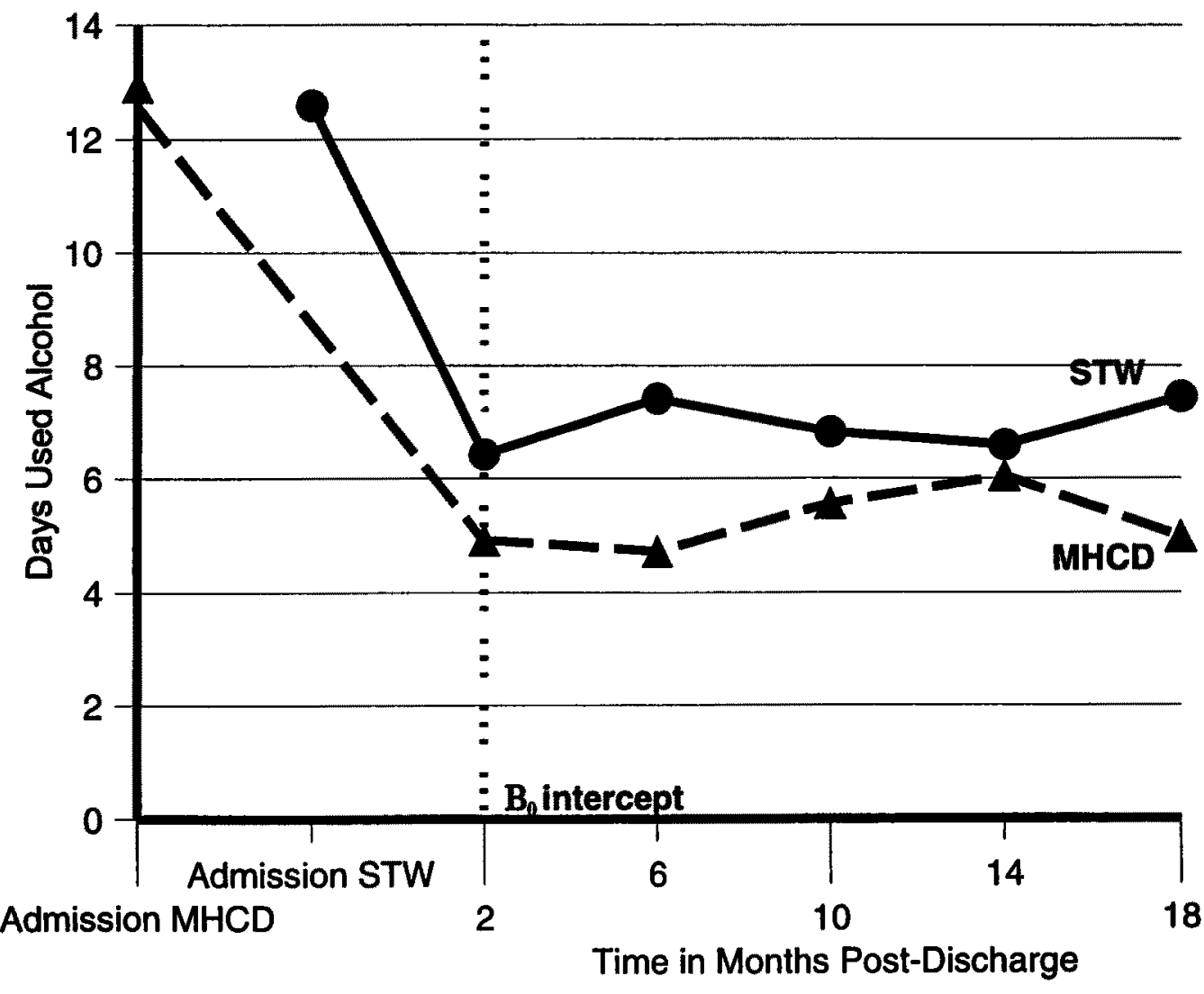

And at level 2 (people): $\quad \beta_{0 i}=\gamma_{00}+\mu_{0 i}$,

$$
\begin{aligned}
& \beta_{1 i}=\gamma_{10}+\mu_{1 i}, \text { and } \\
& \beta_{2 i}=\gamma_{20}+\mu_{2 i} .
\end{aligned}
$$

At level 1, each variable has been subscripted to represent the observation for person $i$ at time $t$. This represents the nested nature of the data. The parameters $\beta_{0 i}, \beta_{l i}$, and $\beta_{2 i}$ each are assigned subscripts $i$ indicating that they are uniquely estimated for each person $i$. Then, at level 2, each parameter is modeled as a function of the overall effect $\left(\gamma_{00}, \gamma_{10}\right.$, and $\gamma_{20}$, respectively), as well as an error term $\left(\mu_{i j}, \mu_{i i}\right.$, and $\mu_{2 i}$, respectively). It is these error terms that capture and account for the unique effects of each person on the intercept and growth parameters. ${ }^{54-56}$

Treatment was implemented at the level of the participant (e.g., each person either received or did not receive the treatment), and, therefore, the treatment effect is modeled at level 2:

$$
\beta_{o i}=\gamma_{00}+\gamma_{01} \text { treatment }_{i}+\mu_{O i}
$$




$$
\begin{gathered}
\beta_{I i}=\gamma_{10}+\gamma_{11} \text { treatment }_{i}+\mu_{I i} \text {, and } \\
\beta_{2 i}=\gamma_{20}+\gamma_{21} \text { treatment }_{i}+\mu_{2 i} \text {. }
\end{gathered}
$$

The effect of the treatment on the intercept is captured by $\gamma_{01}$, the effect on the change during the first time interval by $\gamma_{11}$, and the change on the second time interval by $\gamma_{21}$.

The advantage of the multilevel framework is that it accounts for unbalanced and incomplete data in estimating the parameters. For example, during the second time interval, some subjects were measured at as many as five time points four months apart, while other subjects were measured at three time points at unequal intervals. Rather than deleting data or modifying the time intervals as would be required by a traditional repeated measures ANOVA, the multilevel model uses all of the data in their original form. The key is that the model is specified as a function of time, and the estimation essentially weights for the number of observations per person, so those people who were measured less frequently contribute less to the overall estimates.

An alternate way of appreciating the advantage of the multilevel model is to observe that the sources of error in the models are at level $2\left(\mu_{0 i}, \mu_{i i}\right.$, and $\left.\mu_{2 i}\right)$. This allows the treatment effect, which was defined at level 2 , to be assessed relative to the variability in the errors at level 2 . These level 2 errors are more likely to be independent than the error in a single-level model (such as equation 2) because the level 2 errors (in equation 4 ) represented differences between people. Thus, the effect of level 2 predictors (such as treatment) can be reported in terms of variation explained at level 2 of the model. Furthermore, it recognized that the degrees of freedom used as a basis for assessing the treatment effect should be based on the number of subjects, not the total number of observations. Thus, the standard errors for the treatment effect reflect the level at which the treatment was implemented. This, in turn, reduces the artificial deflation in the standard error term caused by multiple time points.

The multilevel framework can easily accommodate covariates that vary with respect to time (such as whether a person was incarcerated during the month prior to each interview) by incorporating them into the level 1 model. Covariates that are invariant with respect to time (such as whether a person was diagnosed with alcohol dependence or abuse) are incorporated into level 2 of the model. The multilevel model can also account for the effects of level 2 predictors on any level 1 effect. For example, the model can specify the effect of an alcoholism diagnosis on the rate of change in days of alcohol use during the first time interval. In this way, the multilevel model accounts for all of the factors of the traditional split-plot and repeated measure designs while allowing for more flexible model specification and estimation based on unbalanced data.

The final multilevel model for days of alcohol use for person $i$ and time $t$ was the following:

At level 1:

$\log \left(\mathrm{DAU}_{i t}\right)=\beta_{0 i}+\beta_{l i}$ time from admittance to 2 months postdischarge $_{i t}+$

$\beta_{2 i}$ time 2 months postdischarge through end of study ${ }_{i t}+$

$\beta_{3 i}$ participation in self-help $_{i t}+\beta_{4 i}$ incarceration $_{i t}+$

$\beta_{s i}$ support for sobriety $_{i t}$,

And at level 2:

$\beta_{0 i}=\gamma_{00}+\gamma_{01}$ treatment $_{i}+\gamma_{02}$ schizophrenia diagnosis $_{i}+$

$\gamma_{03}$ alcohol diagnosis $s_{i}+\gamma_{04}$ intentions to stay sober ${ }_{i}+$

$\gamma_{05}$ family involvement in treatment ${ }_{i}+\mu_{0 i}$,

$\beta_{l i}=\gamma_{10}+\gamma_{11}$ treatment $t_{i}+\gamma_{12}$ intentions to go to self-help $p_{i}+$

$\gamma_{13}$ family involvement in treatment ${ }_{i}+\mu_{l i}$,

$\beta_{2 i}=\gamma_{20}+\gamma_{21}$ family involvement in treatment ${ }_{i}+\mu_{2 i}$,

$\beta_{3 i}=\gamma_{30}+\gamma_{31}$ intentions to stay sober $_{i}$,

$\beta_{4 i}=\gamma_{40}+\gamma_{41}$ gender $_{i}+\gamma_{42}$ alcohol diagnosis ${ }_{i}+\gamma_{43}$ intentions 


$$
\begin{aligned}
& \text { to stay sober } \\
& \beta_{5 i}=\gamma_{50}+\gamma_{51} \text { treatment }_{i}+\gamma_{52} \text { alcohol diagnosis }_{i}+\gamma_{53} \text { family } \\
& \text { involvement in treatment }
\end{aligned}
$$

At level 2, each level 1 parameter is modeled as a function of individual characteristics (diagnosis, gender, family involvement), the proximal treatment outcomes (motivation), and/or posttreatment environmental characteristics (self-help use, incarceration, and social support for sobriety). In this case, we observe that the intercept $\beta_{o i}$ is a function of treatment, schizophrenia diagnosis, diagnosis of alcohol abuse or dependence, intentions to stay sober, and family involvement in treatment.

The intercept in the level 1 model, $\beta_{D_{i}}$, was defined to represent the person's days of alcohol use at two months after discharge from the treatment or control condition, thus allowing differences in the treatment and control groups at two months postdischarge to be identified (see Bryk and Raudenbush, ${ }^{33}$ pages $25-29$, for a discussion of centering; centering has no effect on the interpretation of the other regression coefficients, $\beta_{i i}, \beta_{2 i}, \beta_{3 i} i$, and $\beta_{4 i}$, only on $\beta_{0 i}$ ). Nonrandomly, time varying covariates have been included to indicate whether the person participated in a self-help process (such as $\mathrm{AA}$ ), was incarcerated during the 30 days previous to the interview, and the amount of support for sobriety the person reported. The effects of the latter three covariates are fixed across people and do not have an error term specified at level 2 (there is no $\mu_{3 i}, \mu_{4 i}, \mu_{5 i}$ ).

The model was tested in the hierarchical generalized linear model (HGLM) routine of Bryk, Raudenbush, and Congdon. ${ }^{56} \mathrm{~A}$ Poisson sampling model was used with a log link function, which is appropriate for count data. Equal exposure or opportunity for occurrence (number of days) was specified. The first step in testing this model was to establish the unconditional model, which is the level 1 model with only the time variables included (e.g., with no level 2 predictors or other level 1 predictors, equation 3). Other level 1 predictors were tested in the model using a step-up strategy guided by the theoretical model. ${ }^{53}$ Once all level 1 predictors had been entered, level 2 predictors were entered. Again, a step-up strategy guided by the theoretical model was used with treatment condition tested first, followed by individual characteristic variables, the proximal treatment outcome variables, and then the posttreatment environmental variables.

\section{Measures}

Days of alcohol use was collected in the alcohol and drug section of the ASI and reflected the number of days the participant reported consuming any alcohol (Table 2). Time was measured in months from the first community interview at two months postdischarge to previous (admission) interview and to each subsequent community interviews. ${ }^{53}$ Thus, the base time point in the model applies to two months postdischarge. Data on use of informal self-help groups (e.g., AA and/or NA) in the past 30 days were collected as self-reports in the interviews $(0=$ no attendance, $1=$ had attended). Incarceration in the past 30 days was based on self-reports provided as part of the ASI interview $(0=$ no, $1=$ yes). Support for sobriety was asked during enumeration of participants' social networks. Participants were asked how much help each social network member gave them in staying clean $(1=$ not at all, $5=$ extremely). Mean level of support was calculated across all members of the social network for each participant. Family involvement in treatment was coded based on hospital records to indicate level of involvement by family members during the inpatient treatment $(1=$ not involved, 2 = moderately involved, 3 = involved). Treatment group was coded 0 for STW (control) and 1 for MHCD (experimental). Diagnosis of schizophrenia $(0=$ no, $1=y e s)$, diagnosis of alcohol dependence or abuse $(0=$ no, $1=$ yes $)$, and gender $(0=$ female, $1=$ male $)$ were also coded as dichotomous variables. Final psychiatric and substance use diagnoses assigned prior to discharge were collected from hospital clinical records and used to create the three diagnosis variables. Two measures of motivation were assessed at discharge. A two-item scale assessed future intentions to stay sober $(1=$ no intentions, $4=$ strong intention; $\alpha=81$ ). The second variable was also from the discharge inter- 


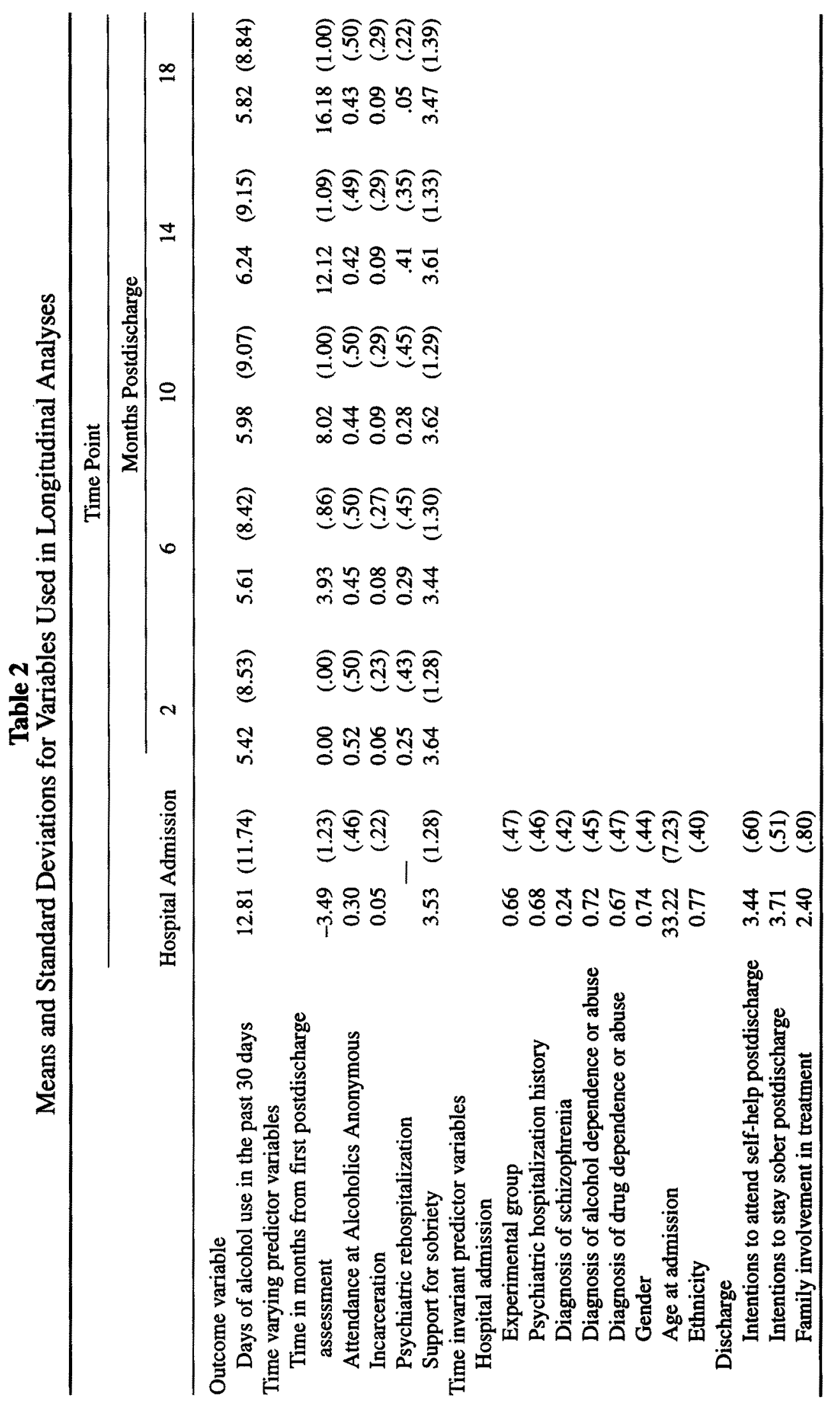


view and asked participants how strong their intentions were to attend self-help groups $(1=$ no intentions, 4 = strong intention). ${ }^{46}$

Other level 1 and level 2 covariates were considered, but none reached the traditional significance level of $p<.05$. These were psychiatric hospitalization during the 30 days prior to each interview $(0=$ no, $1=$ yes), diagnosis of drug dependence or abuse $(0=$ no, $1=$ yes $)$, prior psychiatric hospitalization history $(0=$ none, $1=1$ or more $)$, age at admission, and race/ethnicity $(0=$ white, $1=$ African American).

\section{Results}

The population-average model results, which indicate how a change in the predictor can be expected to affect the overall population mean, are presented in Table 3. Table 3 presents $\beta$ and $e$, which is the event rate or the estimated number of events (days of alcohol use) during time interval. Negative $\beta$ s indicate declines in event rates, and positive $\beta$ s indicate increases in the event rate. The base days of alcohol use at two months postdischarge $\left(\hat{y}_{00}\right)$ was $e^{1.55}$ or estimated at 4.71 . Treatment $\left(\hat{y}_{01}\right)$ reduced the base rate of alcohol use $\left(e^{-.61}\right)$, controlling for other variables in the model $(p<.001)$. In other words, for someone who did not receive the integrated treatment, the expected rate of alcohol use was 4.71 days per month. The expected rate was $4.71 \times .54=2.54$, if the person received the integrated treatment. This constitutes a 54\% reduction in days of use if the person participated in the treatment. Having a diagnosis of schizophrenia $\left(\hat{y}_{02}\right)$ was negatively related to the rate of alcohol use $\left(e^{-.67}\right)$. However, those with an alcohol dependence or abuse diagnosis $\left(\hat{y}_{03}\right)$ had a higher rate of alcohol use $\left(e^{1.13}\right)$. Stronger intentions to stay sober $\left(\hat{y}_{04}\right.$, measured at discharge) reduced the rate of use $\left(e^{-0.26}\right)$. Although not reaching a traditional significant level of $p<.05$, family involvement in inpatient treatment $\left(\hat{y}_{05}\right)$ also reduced the rate of alcohol use $\left(e^{-.15}\right)$.

What these finding indicate is that the rate of alcohol use at two months postdischarge depended on a number of variables. To calculate the effect of parameters on the rate of use, the coefficients are exponentiated $\left(e^{\beta}\right)$ and these values multiplied by the base rate. For example, people with diagnoses of alcoholism who were in the STW group, had low intentions, and no family involvement had the highest rates of use, averaging 14.6 days per month $(4.71 \times 3.10=14.6$; all other parameters have 0 values and drop out of the equation). Similar persons in the MHCD program had an average rate of $7.88(4.71 \times 3.10 \times .54=7.88)$. Thus, the treatment effect for persons with diagnosis of alcoholism and a mental illness other than schizophrenia was to reduce the rate of alcohol use by 6.72 days per month. Among persons with diagnoses of alcoholism, those who also had diagnoses of schizophrenia, received the MHCD treatment, had strong intentions to stay sober, and had high levels of family involvement had a lower rate of 2.66 days of use per month $(4.71 \times 3.10 \times .54 \times .51 \times .77 \times .86=$ 2.66). Among persons without diagnoses of alcoholism, those in the MHCD program had lower rates of use, 2.54 days per month $(4.71 \times .54=2.54)$. The addition of the other characteristics further reduced the rate of use for this latter group to an average of .86 days per month $(4.71 \times .54 \times .51 \times .77 \times$ $.86=.86)$.

In the first time period, the rate of alcohol use declined from admission to two months after discharge by .74 days per month. MHCD treatment further accelerated the decline in the rate of use, as did strong intentions to go to self-help and high levels of family involvement in treatment. In the second time period, the rate of change in days of alcohol use from two months postdischarge through the duration of the study was estimated at 1.01 days. This rate of change did not reach the traditional level for statistical significance $(p<.05)$. Note that there was no effect of treatment on the rate of change in days of alcohol use after two months postdischarge but that the rate remained relatively stable, as do the differences between the groups.

Three additional time-varying predictors affected the changes in the rate of alcohol use. The rate of alcohol use at any given time point was less for persons who had attended meetings of a self-help group during the previous month. Strong intentions at discharge to stay $\operatorname{sober}\left(\hat{y}_{31}\right)$ offset the effect of 
Results of Hierarchical Linear Modeling (population average model)

Fixed Effects

Intercept for $\beta_{0}$

Mean days used alcohol at 2 months

postdischarge $\beta_{\infty}$

Treatment effect $\beta_{01}$

Schizophrenia diagnosis $\beta_{02}$

Alcoholism diagnosis $\beta_{03}$

Intentions to stay sober $\beta_{04}$

Family involvement in treatment $\beta_{05}$

Slope for 2 months postdischarge to admission $\beta_{1}$

Intercept $\beta_{10}$

Treatment effect $\beta_{11}$

Intentions to go to self-help $\beta_{12}$

Family involvement in treatment $\beta_{13}$

Slope for 2 months to 18 months postdischarge $\beta_{2}$

Intercept $\beta_{20}$

Family involvement in treatment $\beta_{21}$

Slope for self-help attendance $\beta_{3}$

Intercept $\beta_{30}$

Intentions to stay sober $\beta_{31}$

Slope for incarceration $\beta_{4}$

Intercept $\beta_{40}$

Gender $\beta_{41}$

Alcoholism diagnosis $\beta_{42}$

Intentions to stay sober $\beta_{43}$

Slope for support for sobriety

Intercept $\beta_{50}$

Treatment effect $\beta_{51}$

Alcoholism diagnosis $\beta_{52}$

Family involvement in treatment $\beta_{53}$
Coefficient $\beta$ Standard $T \quad p$

$\left(e^{\beta}\right) \quad$ Error Ratio Value 
treatment for mental illness and substance use reduced the observed impact of social network support for sobriety. In other words, treatment overcame the lack of social support for sobriety. The final model accounted for $18.8 \%$ of the variance in the intercept when the final level 1 model with level 2 predictors was compared to the final level 1 model without level 2 predictors.

\section{Discussion}

The theoretical model for dual diagnosis treatment suggested that the treatment effects would be influenced by individual characteristics, the proximal treatment outcomes, and what happens when the individual returns to the community in terms of social support and use of formal and informal services. In this study, differential longitudinal outcomes were affected by variables suggested by the theoretical model. ${ }^{23,24}$ Participants' use of alcohol was determined, in part, by their diagnoses, immediate treatment outcomes (intentions), social support, and incarceration. Status on each of these variables was associated with increases or decreases in the number of days on which alcohol was used. The long-term effects of treatment were not uniform. Failure to consider the effects of specific individual characteristics (such as diagnosis), whether treatment produced the desired immediate changes in the individual, and posttreatment experiences may be responsible for the lack of treatment effects in other studies.

The proximal outcome of treatment-motivation for change-was an important covariate. Persons in the MHCD program reported significantly stronger intentions to remain sober and attend self-help groups when they returned to the community. ${ }^{46}$ Stronger intentions at discharge to stay sober predicted lower rates of alcohol use at two months postdischarge, and intentions to attend self-help groups predicted greater rates of change from admission to two months postdischarge. These findings suggested that the MHCD program prepared participants to deal with their substance use problems. It helped participants to be ready to change. Those participants who received the dual treatment and reached the critical point of change, indicated by their strong intentions to stay sober, reduced their alcohol consumption more than those who did not receive the treatment regardless of their lèvel of intentions.

The findings also point out the potential for posttreatment experiences to affect the observed outcomes. Self-help attendance reduced the rate of alcohol use. It was particularly effective when motivation to stay sober was low, such as was the case for the control group. Incarceration also resulted in fewer days of alcohol use. Number of days incarcerated was not collected, and this limits explanations as to why incarceration resulted in fewer days of alcohol use. It is possible that incarceration reduced the number of days during which alcohol could be accessed and this resulted in artificially low scores. Greater family involvement while the individual was hospitalized was associated with stronger levels of support for sobriety and magnified the effect of such support in decreasing alcohol use. However, treatment in the MHCD program nearly offset the effect of such support. In contrast, social support for sobriety was important to reducing the rate of use for those in the control condition. Again, this suggests that the MHCD program affected some change intrinsic to the person. In the absence of such a change, an external force, such as self-help attendance or social support for sobriety, was needed to reduce alcohol use.

\section{Limitations}

As in other studies of this type, this one has limitations due to methodology and resource constraints. The outcome measure is based on self-reports and suffers from the biases inherent in all self-reports. Corroboration from another source, whether biochemical, clinical ratings, proxy reports, or another instrument, ${ }^{13}$ would strengthen confidence in the level of alcohol use by participants. Self-selection to attend self-help posthospitalization is another potential limiting factor. The current study did not have the means to determine whether attenders were different from nonattenders in important but unmeasured ways. This is an underdeveloped area within SMI-SD 
treatment that warrants greater investigation. Additionally, the measure of self-help attendance used in this study is weak and did not permit the quality of participation to be assessed. However, the study does point to a potentially powerful community asset-mutual support-for persons with serious mental illness and substance use.

\section{Implications for Behavioral Health Services}

The value of an inpatient program that simultaneously treats both serious mental illness and substance use is its ability to give the consumer a safe, structured environment in which education, counseling, and participation in self-help can lead to changes in motivation to deal with the substance use problem. The results of this study indicated that dual diagnosis programs are valuable and can establish a basis for long-term change and improved community functioning. The study showed that support for sobriety is important to long-term outcomes and that the treatment effects for such programs can overcome lack of support for sobriety. One means of providing such support appears to be through self-help groups, such as AA. Although much of the literature on dual treatment programs identifies families and social networks as key to posttreatment sobriety, ${ }^{3,37,38,57}$ their roles in ongoing aftercare treatment have not been emphasized. The results of this study point to the need for ongoing careful assessment of the characteristics of the individual's environment and social resources as part of effective mental health care.

The findings of this study suggest that as mental health systems move to managed care packages, inpatient treatment programs for substance use and serious mental illness can be a valuable component. These programs should not be treated, however, as a separate component in an array but as a first step in a treatment process. ${ }^{58}$ The treatment process should include assessments of the individual's motivation to change and willingness to continue with behaviors, such as attending AA, that are established while in the hospital. The treatment process should develop with the consumer clear plans for achieving his or her goals after leaving the hospital, including the use of formal services and self-help groups, and emphasize individual planning. ${ }^{58}$ It is important for the treatment process to help the consumer develop links with community self-help groups. Early connection with community groups should be initiated while the consumer is still in the integrated treatment program and should focus on developing a relationship with a sponsor. Postdischarge support for self-help attendance should be part of the aftercare provided.

The posthospitalization treatment process should support and encourage attendance at self-help groups and continue to monitor, with the consumer, his or her motivation to stay sober and psychologically healthy. Maintaining the individual's motivation will require different strategies than have traditionally been used for aftercare services. Assessment with the consumer of his or her environment, social network/support system, and community for ongoing support and threats to sobriety will be essential. Mental health services should focus therapeutic efforts on helping consumers to gain skills in managing their mental illness, maintaining sobriety, and achieving goals through flexible, accessible, personalized services. ${ }^{58}$ Although person-centered approaches are not the general practice, a focus on the goals of the person throughout the treatment process will help develop the internal motivation to maintain gains in sobriety achieved through integrated treatment programs.

\section{Acknowledgments}

The Michigan Department of Community Health (formerly the Department of Mental Health) received funding for this study from the National Institute of Mental Health under its Enhancing Research in State Mental Health Agencies initiative (MH46307). The study is a collaborative effort between the Michigan Department of Community Health's Service Research Unit and Northville Psychiatric Hospital, Detroit-Wayne Community Mental Health Board, Michigan State University, Wayne State University, and the University of Michigan. The views and opinions expressed by the 


\section{References}

1. Minkoff K: Program components of a comprehension integrated care system for serious mentally ill patients with substance disorders. New Directions for Mental Health Services 1991; 50:13-27.

2. Ridgely MS: Creating integrated programs for severely mentally ill persons with substance abuse disorders. New Directions for Mental Health Services 1991; 50:29-42.

3. Mueser KT, Drake RE, Miles KM: The course and treatment of substance use disorder in persons with severe mental illness. In NIDA Monograph: Treatment of Drug-Dependent Individuals with Comorbid Mental Disorders (RM 172). Washington DC: NIDA, 1997, pp. 86-109.4.

4. Osher FC, Kofoed LL: Treatment of patients with psychiatric and psychoactive substance abuse disorders. Hospital and Community Psychi * atry 1989; 40:1025-1030.

5. Drake RE, McLaughlin P, Pepper B, et al.: Dual diagnosis of major mental illness and substance disorder: An overview. New Directions for Mental Health Services 1991; 50:3-12.

6. Lehman AF, Myers CP, Corty E: Assessment and classification of patients with psychiatric and substance abuse syndromes. Hospital and Community Psychiatry 1989; 40:1019-1025.

7. Durell J, Lechtenberg B, Corse S, et al.: Intensive case management of persons with chronic mental illness who abuse substances. Hospital and Community Psychiatry 1993; 44: 415-416, 428.

8. Bond GR, McDonel EC, Miller LD, et al.: Assertive community treatment and reference groups: An evaluation of their effectiveness for young adults with serious mental illness and substance abuse problems. Psychosocial Rehabilitation Journal 1991; 15:31-47.

9. Jerrell JM, Ridgely MS: Evaluating changes in symptoms and functioning of dually diagnosed clients in specialized treatment. Psychiatric Services 1995; 46:233-237.

10. Ridgely MS, Jerrell JM: Analysis of three interventions for substance abuse treatment of severely mentally ill people. Community Mental Healh Journal 1996; 32:561-572.

11. Bartels SJ, Drake RE, Wallach MA: Long-term course of substance use disorders among patients with severe mental illness. Psychiatric Services $1995 ; 46: 248-251$.

12. Drake RE, McHugo GJ, Noordsy DL: Treatment of alcoholism among schizophrenic outpatients: 4year outcomes. American Journal of Psychiatry 1993; 150:328-329.

13. Drake RE, Yovetich NA, Bebout RR, et al.: Integrated treatment for dually diagnosed homeless adults. Journal of Nervous and Mental Disease 1997; 185:298-305.

14. Munsey DF, Galanter M., Lifshutz $H$, et al:: Antecedents, severity of abuse, and response to treatment in substance-abusing schizophrenic individuals. American Journal on Addictions 1992; 1:210-216.

15. Case N: The dual-diagnosis patient in a psychiatric day treatment program: A treatment failure. Joumal of Substance Abuse Treatment 1989; 8:69-73.

16. Lyons JS, McGovern MP: Use of mental health services by dually diagnosed patients. Hospital and Community Psychiatry 1989; 40:1067-1069.

17. Blankertz LE, Cnaan RA: Principles of care for dually diagnosed homeless persons: Findings from a demonstration project. Research on Social Work Practice 1992; 2:448-464.

18. Drake R, Mercer-McFadden C, Mueser K, et al.: Review of integrated mental health and substance abuse treatment for patients with dual disorders. Schizophrenia Bulletin 1998; 24:589-608.

19. Finney JW, Noyes, CA, Coutts, AI, et al.: Evaluating substance abuse treatment process models: I. Change on proximal outcome variables during 12-step and cognitive-behavioral treatment. Joumal of Studies on Alcohol 1998; 59:371-380.

20. Hanson M, Foreman L, Tomlin W, et al. Facilitating problem drinking clients' transition from inpatient to outpatient care. Health \& Social Work 1994; 19:23-28.

21. Bartels SJ, Thomas WN: Lessons from a pilot residential treatment program for people with dual diagnoses of severe mental illness and substance use disorder. Psychosocial Rehabilitation Journal 1991; 15:19-30.

22. Alfs DS, McLellan TA: A day hospital program for dual diagnosis patients in a veteran's medical center. Hospital and Community Psychia* try 1992; 43:241-244.

23. Finney JW, Moos RH: Theory and method in treatment evaluation. Evaluation and Program Planning 1990; 12:307-316.

24. Moos, RH, Finney J, Maude-Griffin P: The social climate of self-help and mutual support groups: Assessing group implementation, process, and outcome. In McCrady B, Miller W (Eds.); Research on Alcoholics Anonymous. New Brunswick, NJ: Rutgers Center of Alcohol Studies, 1993, pp. 251-274.

25. Lehman A: Heterogeneity of person and place: Assessing co-occurring addictive and mental disorders. American Journal of Orthopsychiatry 1996; 66:32-41.

26. Luke $\mathrm{D}$, Mowbray, $\mathrm{CT}$, Klump $\mathrm{K}$, et al: Exploring diversity of dual diagnosis: Utility of cluster analysis for program planning. Journal of Mental Health Administration 1996; 23:260-271.

27. Arndt S, Tyrrell G, Flaum M, et al.: Comorbidity of substance abuse and schizophrenia: The role of pre-morbid adjustment. Psychological Medicine 1992; 22:379-388.

28. Kay SR, Kalathara M, Meinzer AE: Diagnostic and behavioral characteristics of psychiatric patients who abuse substances. Hospital and Community Psychiatry 1989; 40:1062-1064.

29. Osher FL, Drake RE: Reversing a history of unmet needs: Approaches to care for persons with co-occurring addictive and mental disorders. American Joumal of Orthopsychiatry 1996; 66:4-16.

30. Minkoff K: Integrated treatment model for dual diagnosis of psychosis and addiction. Hospital and Community Psychiatry 1989; 40:1031-1036.

31. Caldwell S, White KK: Co-creating a self-help recovery movement. Special issue: Serving persons with dual disorders of mental illness and substance use. Psychosocial Rehabilitation Journal 1991; 15:91-95. 
32. Mueser KT, Noordsy DL: Group treatment for dually diagnosed clients. New Directions for Mental Health Services 1996; 70:33-51.

33. Carey K: Treatment of co-occurring substance abuse and major mental ilness. New Directions for Mental Health Services 1996 ; $70: 19-32$.

34. Woods $\mathrm{J}$ : Incorporating services for chemical dependency problems into clubhouse model programs: A description of two programs. Psychosocial Rehabilitation Journal 1991; 15:107-112.

35. Johnsen E, Herringer LG: A note on the utilization of common support activities and relapse following substance abuse treatment. Joumal of Psychology 1993; 127:73-77.

36. Drake RE, Mueser K, Clark R, et al.: The course, treatment, and outcome of substance disorder in persons with mental illness. American Joumal of Orthopsychiatry 1996; 66:42-51.

37. Carey K: Treatment of substance use disorders and schizophrenia. In Lehman AF, Dixon LB (Eds.): Double Jeopardy: Chronic Mental IIlness and Substance Use Disorders. Chur, Switzerland: Harwood Academic, 1995, pp. 85-108.

38. Drake RE, Noordsy DL, Ackerson T: Integrating mental health and substance treatments for persons with chronic mental disorders: A model. In Lehman AF, Dixon LB (Eds.): Double Jeopardy: Chronic Mental Illness and Substance Use Disorders. Chur, Switzerland: Harwood Academic, 1995, pp. 251-264.

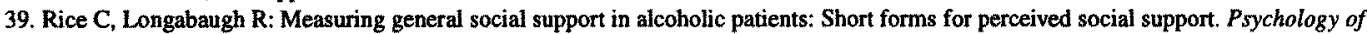
Addictive Behaviors 1996; 10:104-114.

40. Humphreys K, Moos R: Reduced substance-abuse-related health care costs among voluntary participants in Alcoholics Anonymous. Psychiatric Senvices 1996; 47:709-713.

41. Narrow W E, Regier DA, Rae DS, et al.: Use of services by persons with mental and addictive disorders. Archives of General Psychiatry 1993; 50:95-107.

42. Noordsy DL, Schwab B, Fox L, et al.: The role of self-help programs in the rehabilitation of persons with severe mental illness and substance use disorders. Community Mental Health Joumal 1996; 32:71-81.

43. Miller WR: Motivation for treatment: A review with special emphasis on alcoholism. Psychological Bulletin 1985; 98:84-107.

44. Drake RE, Antosca LM, Noordsy DL, et al.: New Hamshire's specialized services for the dually diagnosed. New Directions for Mental Health Services 1991; 50:57-67.

45. Mueser KT, Bellack AS, Blanchard Jf: Cormorbidity of schizophrenia and substance abuse: Implications for treatment. Journal of Consulting and Clinical Psychology 1992; 60:845-856.

46. Herman S, BootsMiller B, Jordan L, et al: Immediate outcomes of substance abuse treatment within a state psychiatric hospital. Journal of Mental Health Administration 1997; 24:126-138.

47. Selzer M, Vinokur A, van Rooijen L: A self-administered short Michigan Alcoholism Screening Test (SMAST). Journal of Alcohol Studies $1975 ; 36: 117-126$.

48. BootsMiller B, Ribisl K, Mowbray C, et al.: Methods of ensuring high follow-up rates: Lessons from a longitudinal study of dual disorder participants. Substance Use and Misuse 1998; 33:2665-2685.

49. McLellan AT, Luborsky L, Woody GE, et al.: An improved diagnostic evaluation instrument for substance abuse patients: The Addiction Severity Index. Journal of Nervous and Mental Disease 1980; 168:26-33.

50. Fureman B, Parikh G, Bragg A, et al.: Addiction Severity Index. Philadelphia: University of Pennsylvania/Veterans Administration Center for Studies on Addiction, 1990

51. Hodgins D, El-Guebaly N: More data on the addiction severity index. Journal of Nervous and Medical Disorders 1992; 180;197-201.

52. Seltzer MH, Frank KA, Bryk AS: The metric matters: The sensitivity of conclusions concerning growth in student achievement to choice of metric. Educational Evaluation and Policy Analysis 1994; 16:41-49.

53. Bryk A, Raudenbush S: Hierarchical Linear Models. Newbury Park, CA: Sage, 1992.

54. Goldstein H: Multilevel Models in Educational and Social Research. London: Oxford University Press, 1995.

55. Longford N: Random Coefficient Models. Oxford: Clarendon, 1993.

56. Bryk A, Raudenbush S, Congdon R. Hierarchical Linear and Nonlinear Modeling with the HLM/2Land HLM/3LPrograms. Chicago: Scientific Software Intemational, 1996.

57. Clark R: Family support for persons with dual disorders. New Directions for Mental Health Services 1996; 70:65-78.

58. Osher F: A vision for the future: Toward a service system responsive to those with co-occurring addiction and mental disorders. American Joumal of Orthopsychiatry 1996; 66:71-76.

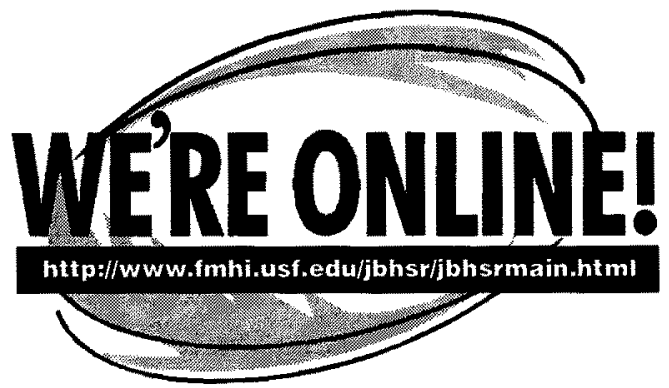

